\title{
Bilateral Optic Disc Swelling with Preserved Visual Function Associated with Giant Cell Arteritis
}

\author{
Mihoko Mochijia Yukari Yamane ${ }^{a} \quad$ Akiko Ishida $^{a}$ Manabu Hondab \\ Yohko Murakawab Masaki Tanito ${ }^{a}$ \\ aDepartment of Ophthalmology, Shimane University Faculty of Medicine, Izumo, Japan; \\ ${ }^{b}$ Department of Rheumatology, Shimane University Faculty of Medicine, Izumo, Japan
}

\section{Keywords}

Bilateral optic disc swelling · Giant cell arteritis · Positron emission tomography

\begin{abstract}
A 68-year-old Japanese man was introduced to our hospital for optic disc swelling (ODS) in his both eyes $(\mathrm{OU})$. Other than floaters in his right eye, he did not report any symptoms including blurred vision, visual field defect, and ocular pain. Light reflex was prompt and complete $\mathrm{OU}$, and critical flicker frequency was within the normal range OU. By fluorescein angiography, hyperfluorescence was detected on optic discs OU; however, no fluorescein leakage or filling defect was observed. By Goldmann perimetry, enlargement of the Mariotte blind spot was revealed OU, while no central scotoma or remarkable visual field defects were detected. By neuroimaging and lumbar puncture, papilledema due to intracranial pressure elevation was denied. Based on the reassessment of fundus findings, narrowing and segmental whitening/sheathing of peripapillary vessels predominantly to arterioles were realized, and systemic arteritis was suspected. Based on the subject age, elevation of erythrocyte sedimentation rate, positron emission tomography findings in the aorta, and MRI findings in temporal arteries, underlying giant cell arteritis (GCA) was diagnosed. After the start of systemic and local steroid therapies, ODS improved OU. Although rare, bilateral ODS with no visual disturbance can occur in patients with GCA. This case emphasizes the importance of careful assessment of ocular findings to reach the correct diagnosis of even a rare cause of ODS.
\end{abstract}

\section{Karger"}




\section{Introduction}

Optic disc swelling (ODS) is caused by impaired axonal transport of optic nerve fibers and develops due to various causes such as pressure, ischemia, metabolic disorders, inflammation, and poisoning $[1,2]$. ODS with preserved visual function differs between bilateral and unilateral cases. Bilateral ODS with preserved visual function is most often a result of papilledema due to intracranial pressure (ICP) elevation [1,3]. Further differential diagnosis is required when papilledema is ruled out, although such condition is rare $[1,4]$.

Giant cell arteritis (GCA) is a systemic granulomatous vasculitis, affecting people over 50 years of age. It has a predilection for the aorta and its major branches. GCA may compromise blood supply to eyes by affecting the ophthalmic artery and has the potential to cause irreversible visual loss due to anterior ischemic optic neuropathy (AION) [5, 6]. Here, we report a case of bilateral ODS without visual loss who was initially suspected of papilledema and was finally diagnosed with ODS associated with GCA.

\section{Case Report}

A 68-year-old Japanese man visited his local ophthalmologist for seeing floaters in his right eye (OD) for a month. His systemic and ocular medical histories included systemic hypertension, senile cataract in his both eyes (OU), and macular degeneration (suspected of macular telangiectasia or age-related macular degeneration) OU. His best-corrected visual acuity (BCVA) of 0.7 OD and 0.3 in his left eye (OS) was equivalent to his previous visit to the local hospital OU. He was introduced to our hospital because of remarkable ODS OU. At the initial visit to our hospital, BCVA was $0.6 \mathrm{OD}$ and $0.4 \mathrm{OS}$, and intraocular pressure was $8 \mathrm{~mm}$ $\mathrm{Hg}$ OD and $10 \mathrm{~mm} \mathrm{Hg} \mathrm{OS.} \mathrm{Light} \mathrm{reflex} \mathrm{was} \mathrm{prompt} \mathrm{and} \mathrm{complete} \mathrm{OU,} \mathrm{and} \mathrm{critical} \mathrm{flicker}$ frequency was within the normal range $(34-38 \mathrm{~Hz})$ OU. Other than floaters OD, he did not report any symptoms including blurred vision, visual field defect, and ocular pain. By funduscopy (Fig. 1a, b) and optical coherence tomography (RS-3000 Advance 2; Nidek, Gamagori, Japan) (Fig. 1c, d), ODS and peripapillary retinal hemorrhage OU were observed. Macular optical coherence tomography scans showed intraretinal cystic spaces and retinal pigment epithelial irregularity OU (Fig. 1e, f), corresponding to the previously determined macular lesions; however, no acute retinal lesion was detected OU. By fluorescein angiography (Heidelberg Retina Angiograph 2; Heidelberg Engineering, Dossenheim, Germany), hyperfluorescence was detected on optic discs OU; however, no filling defect of optic discs or no fluorescein leakage in the macular regions was observed (Fig. 2a-d). By Goldmann perimetry, enlargement of the Mariotte blind spot was revealed OU, while no central scotoma or remarkable visual field defects were detected (Fig. 2e, f). His body temperature was $36.4^{\circ} \mathrm{C}$, and blood pressure was 159/98 mm Hg. By blood testing, elevations of C-reactive protein $(2.69 \mathrm{mg} / \mathrm{dL}$; normal range $<0.14 \mathrm{mg} / \mathrm{dL})$ and erythrocyte sedimentation rate (ESR) (94 $\mathrm{mm} / \mathrm{h}$; normal range $1-10 \mathrm{~mm} / \mathrm{h}$ ) were reported. Overall, since the findings did not support the presence of optic neuritis/neuropathy, papilledema due to ICP elevation was suspected, and he was introduced to the neurology department.

At the neurology department, by lumbar puncture performed in the left lateral decubitus position, ICP was normal $\left(16 \mathrm{~cm} \mathrm{H}_{2} \mathrm{O}\right)$, and neither abnormal increase of protein and glucose levels nor cells were reported in the cerebrospinal fluid specimen. By head CT scan (Fig. 3a) and magnetic resonance venography (Fig. 3b), no intracranial lesions or venous occlusion that can cause papilledema was detected. Blood testing revealed no evidence of thrombosis predisposition. No temporal artery tenderness existed, and no temporal artery thickening or halo signs were presented by ultrasound sonography.

\section{Karger'}




\section{Case Reports in Ophthalmology}
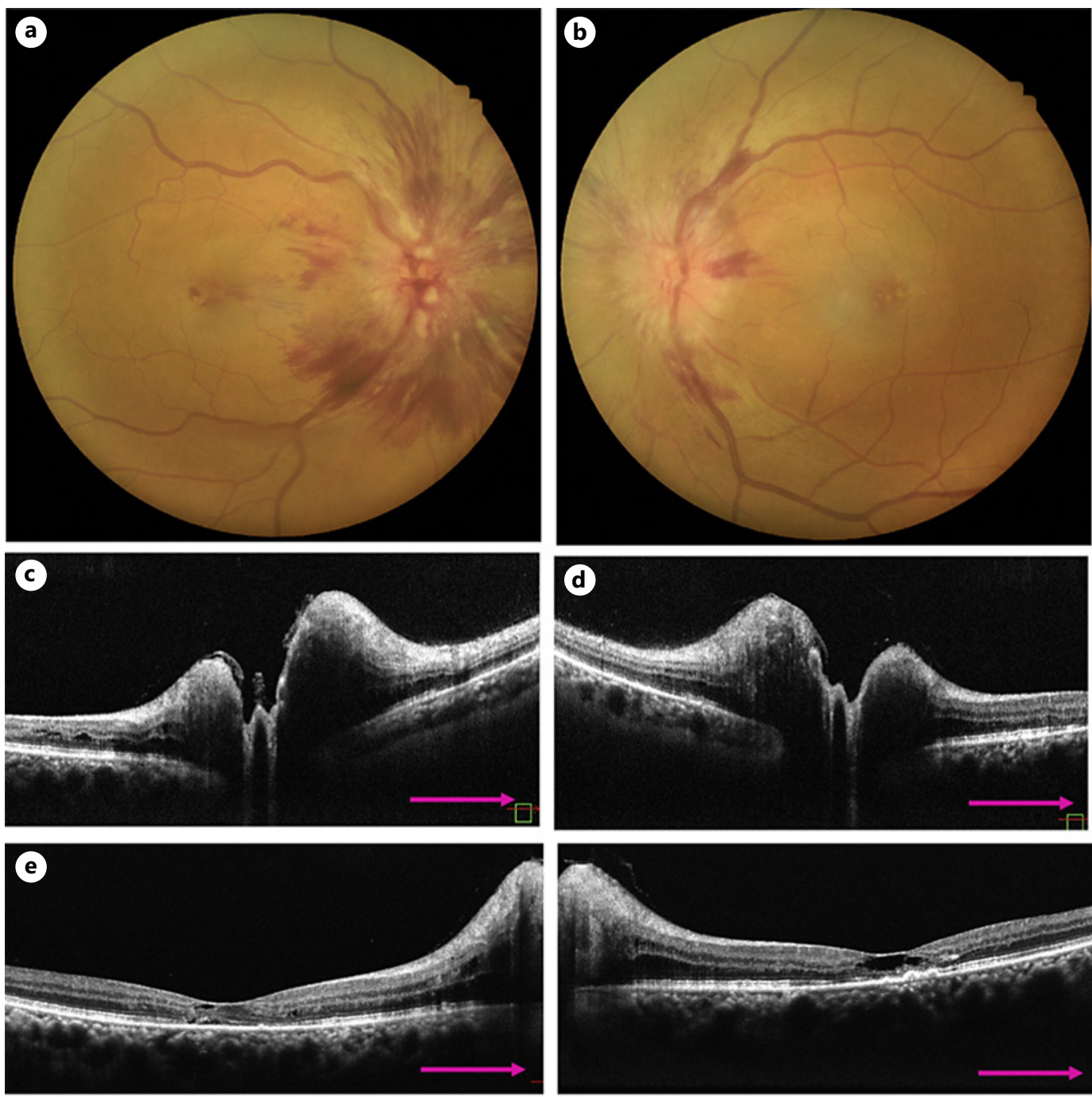

Right eye

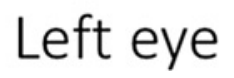

Fig. 1. Fundus photographs (a, b) and OCT scans at the optic nerve head (c, d) and macula (e, f) of the right $(\mathbf{a}, \mathbf{c}, \mathbf{e})$ and left $(\mathbf{b}, \mathbf{d}, \mathbf{f})$ eyes at the initial visit. Fundus photographs $(\mathbf{a}, \mathbf{b})$ and OCT scans at the optic nerve head $(\mathbf{c}, \mathbf{d})$ reveal optic disc swelling with retinal hemorrhage in his both eyes. Macular OCT scans show intraretinal cystic space and retinal pigment epithelial irregularity in both eyes (e, f) that suggests the previously determined macular lesions; no acute retinal lesion is seen in macular scans. Pink arrows indicate OCT scan directions. OCT, optical coherence tomography.

By reviewing the fundus photographs at the clinical conference of the ophthalmology department, narrowing and segmental whitening/sheathing of peripapillary vessels predominantly to arterioles and relative sparing of venules were realized (Fig. 1a, b). At this timing, he reported a mild headache around temporal and occipital regions. By additional blood testing, blood interleukin 6 level $(9.1 \mathrm{pg} / \mathrm{mL}$; normal range $\leq 4 \mathrm{pg} / \mathrm{mL})$ was elevated; while auto-antibodies including proteinase 3 -antineutrophil cytoplasmic antibody (ANCA) and myeloperoxidase (MPO)-ANCA that associate with small vessel vasculitis, anti-Bartonella antibody that associate with cat-scratch disease, and anti-aquaporin 4 antibody that associate with neuromyelitis optica, IgG4 antibody that associate with IgG4-related disease, serologic 


\section{Case Reports in Ophthalmology}
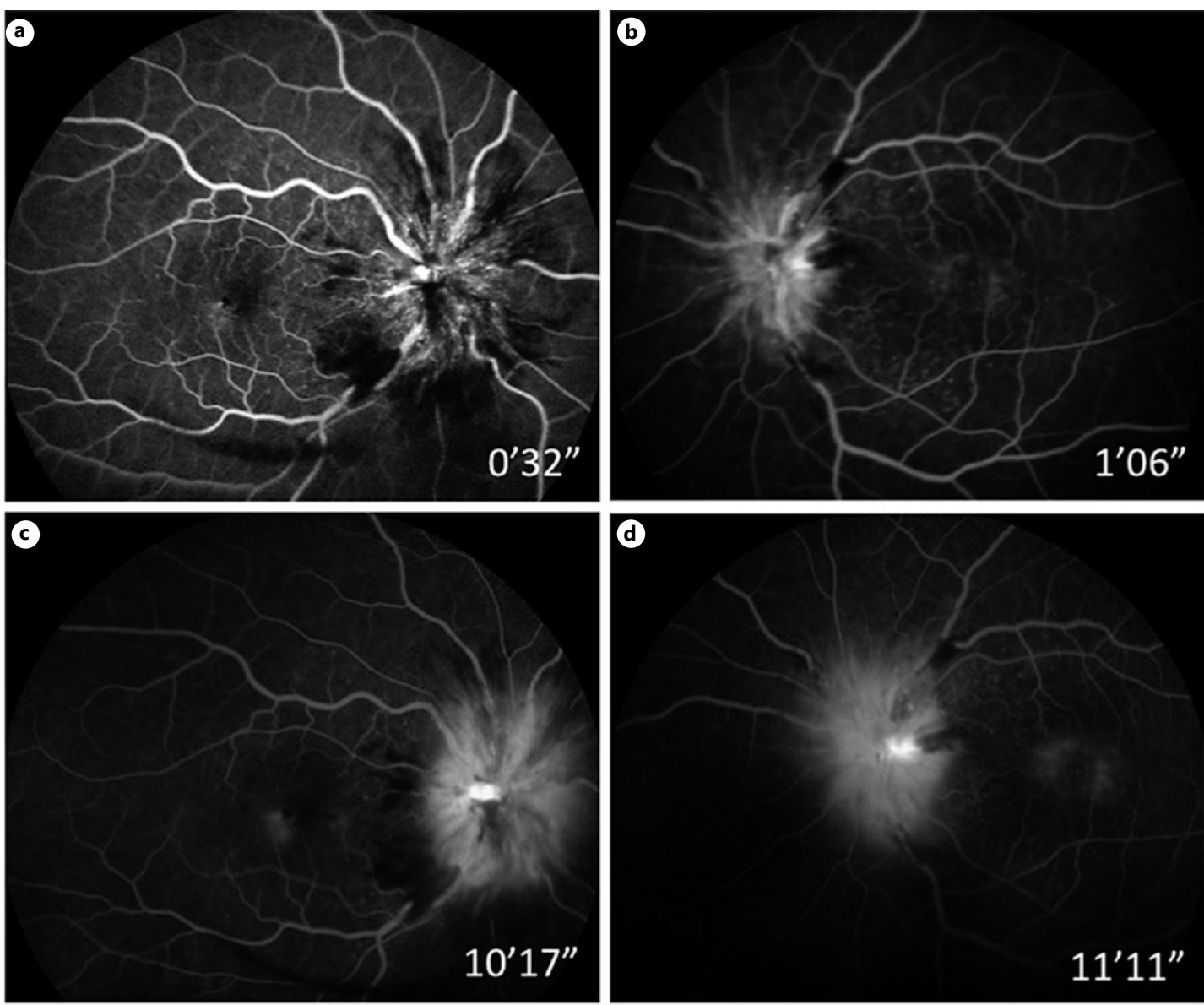

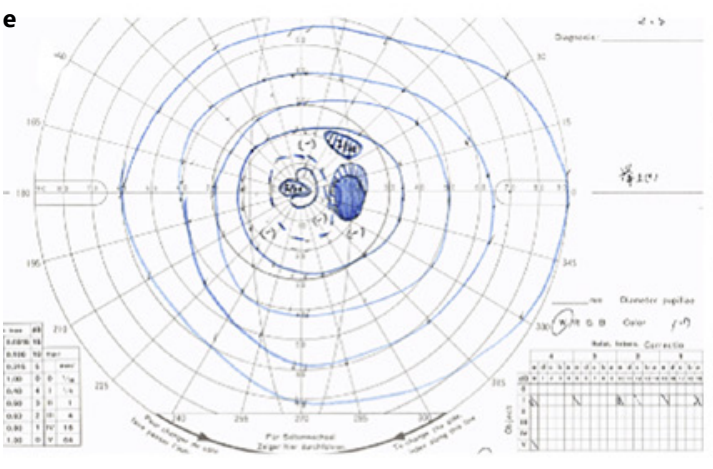

Right eye

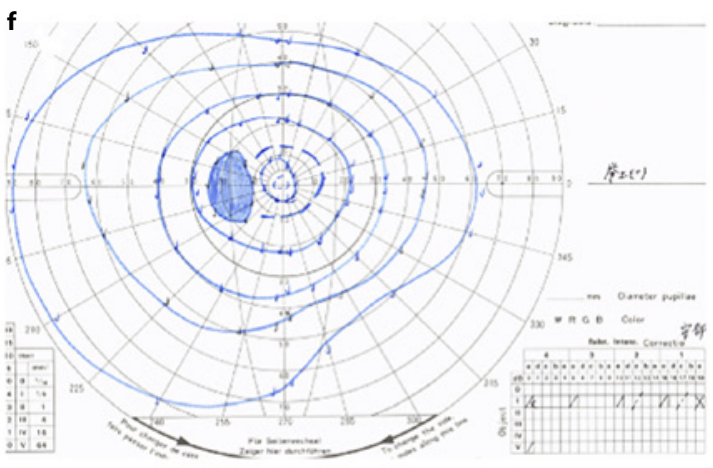

Left eye

Fig. 2. Early (a, b) and late (c, d) phases of fundus fluorescein angiography and Goldmann visual field (e, f) of the right (a, c, e) and left ( $\mathbf{b}, \mathbf{d}, \mathbf{f})$ eyes at the initial visit. a-d Hyperfluorescence corresponding to optic disc swelling is seen; no filling defect of the optic nerve head is observed. e, $\mathbf{f}$ Goldmann visual field reveals enlargement of the Mariotte blind spot with no central scotoma in his both eyes.

test for syphilis, and HLA-DR antigen that associate with Vogt-Koyanagi-Harada disease were all negative or within normal limit. Based on the fundus findings and elevation of ESR, large vessel arteritis was suspected, and he was introduced to the rheumatology department.

By PET, abnormal accumulation of FDG was revealed throughout the aorta including thoracic, abdominal (Fig. 4a), and left common iliac arteries. By CT, enlargement of the 


\section{Case Reports in Ophthalmology}
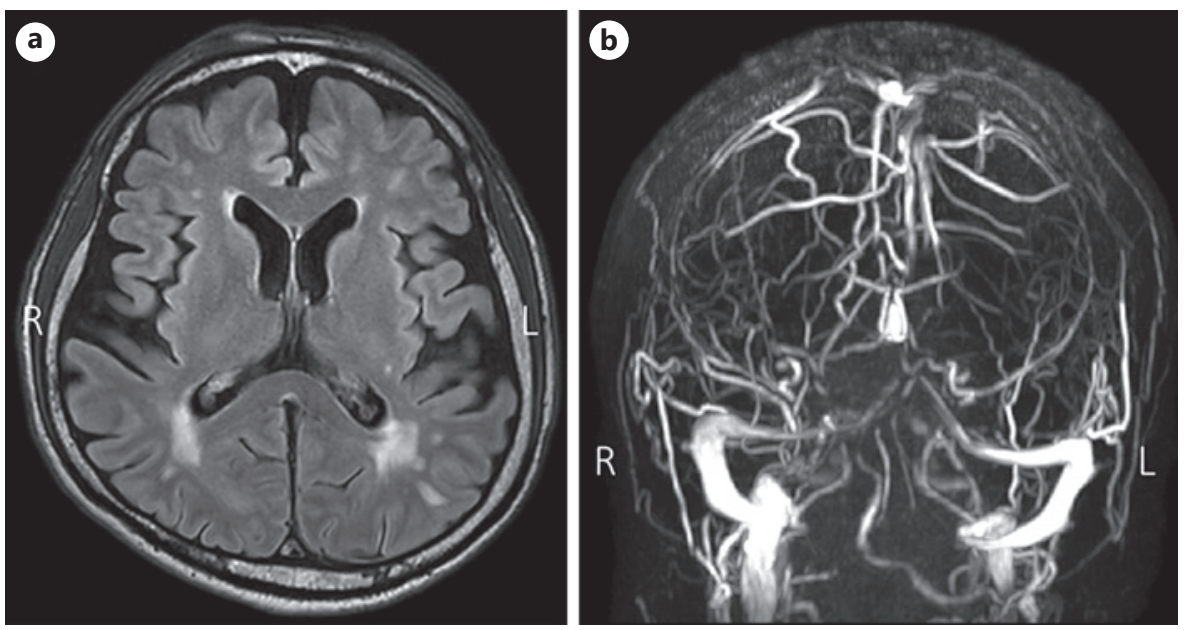

Fig. 3. Brain CT scan (a) and MRV image (b). a, b No intracranial lesions or venous occlusion that can cause papilledema is seen. MRV, magnetic resonance venography.

Fig. 4. Abdominal PET scan (a) and CT scan with contrast (b). Abnormal accumulation of FDG in the abdominal aorta (a, arrow) and enlargement of the aorta (b, arrow heads) suggest abdominal aortic vasculitis. PET, positron emission tomography.
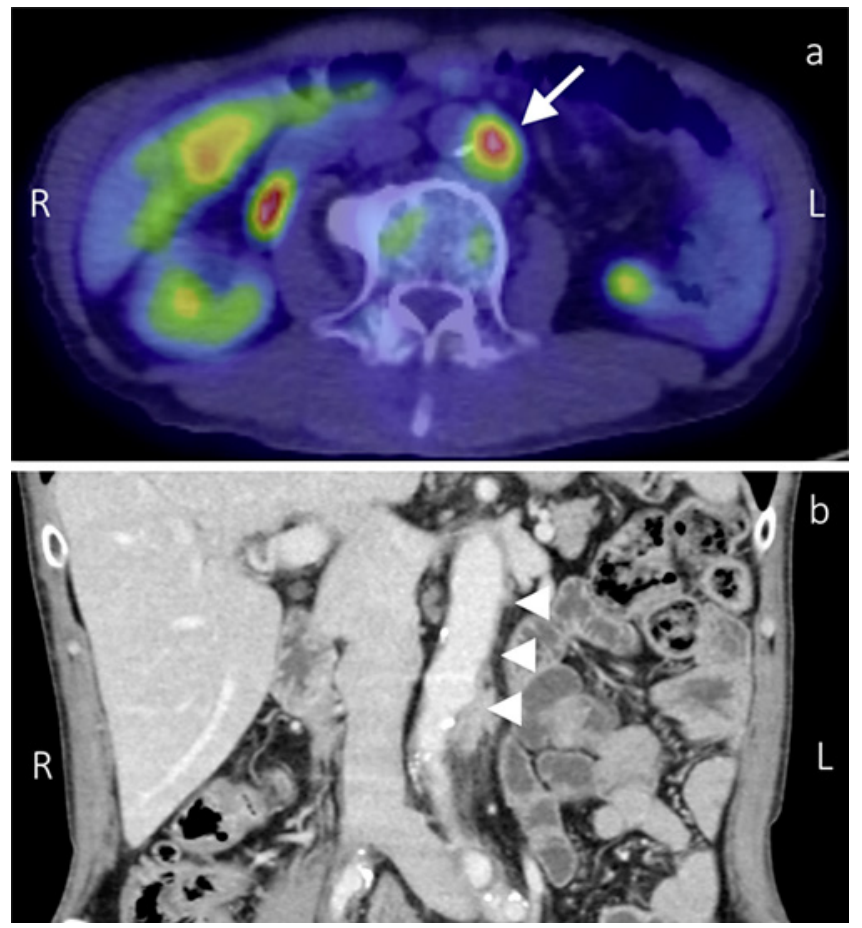

abdominal aorta (Fig. 4b) was seen in the entire abdominal aorta. By contrast MRI, enhancement of superficial temporal arteries in the right (Fig. 5a) and left (Fig. 5b) sides suggests temporal arteritis in both sides. On the same session, there was no enhancement of optic nerve sheath (Fig. 5c, d). The evidence suggests the presence of large vessel arteritis, and he fulfilled the 1990 American College of Rheumatology criteria for GCA (i.e., age >50 years old, elevated ESR, and newly developed headache) [7]. Age of 50 years and involvement of temporal arteries also were supportive findings of GCA rather than Takayasu disease. No findings suggestive of Behçet disease including oral aphtha, genital ulcer, and erythema nodosum and serous uveitis were observed. He was treated with a steroid pulse regimen 


\section{Case Reports in Ophthalmology}
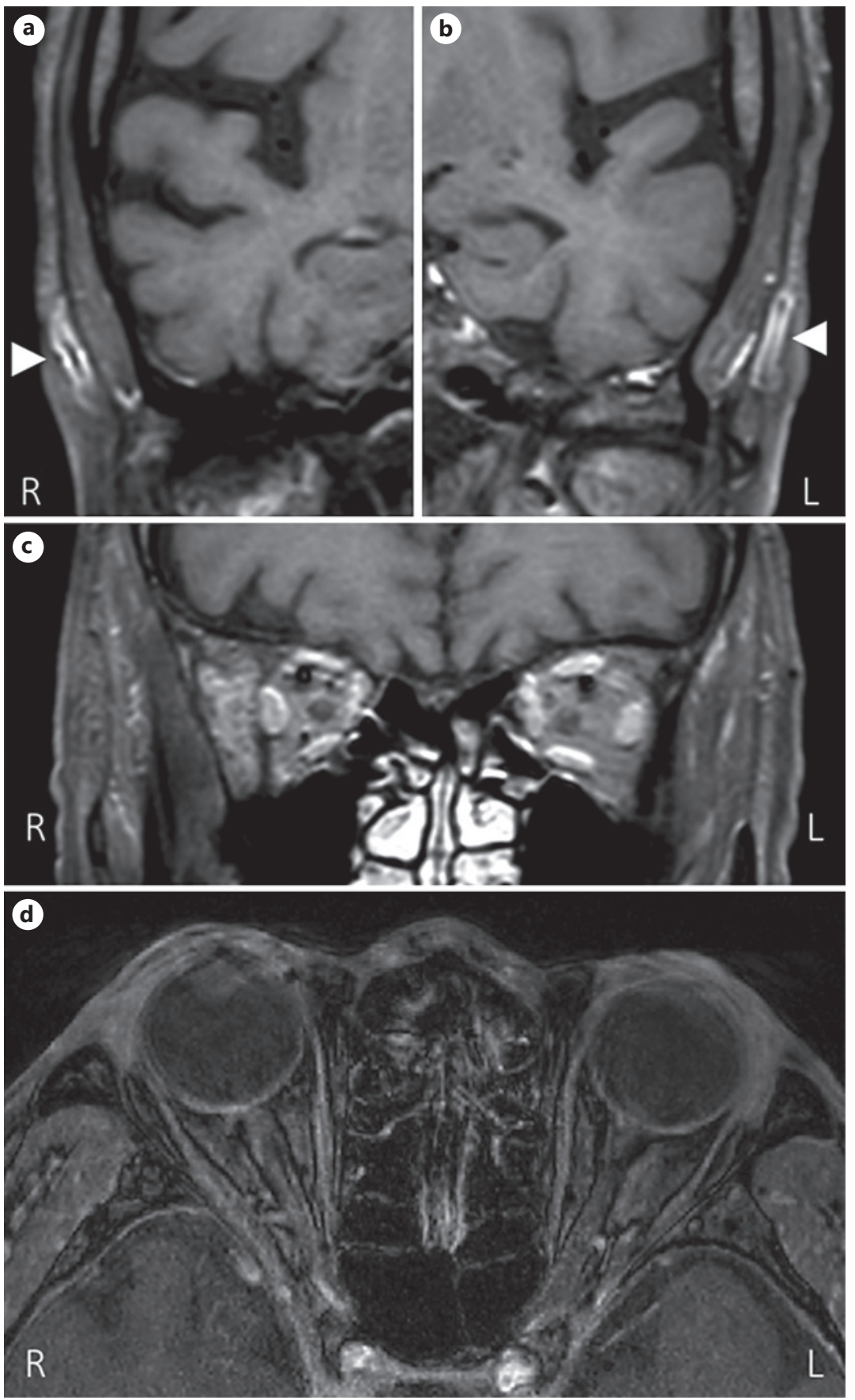

Fig. 5. Gadolinium-enhanced MRI of the head (a, b) and orbit (c, d). Enhancement of superficial temporal arteries in the right (a) and left (b) sides by MRI suggests temporal arteritis. Enhancement of superior optic nerves sheath is not seen in coronal (c) and axial (d) orbital scans.

(methylprednisolone $500 \mathrm{mg} /$ day for 3 days) followed by oral prednisolone ( $50 \mathrm{mg} /$ day) and tapering. For local therapy, triamcinolone $(20 \mathrm{mg}$ ) (MaQaid; Wakamoto Pharmaceutical, Tokyo, Japan) was injected into sub-Tenon space OU. During the hospitalization, blood pressure was at the highest $159 / 98 \mathrm{~mm} \mathrm{Hg}$. Four months after the start of steroid pulse therapy, C-reactive protein $(0.63 \mathrm{mg} / \mathrm{dL})$ and ESR $(29 \mathrm{~mm} / \mathrm{h})$ reduced and headache disap- 

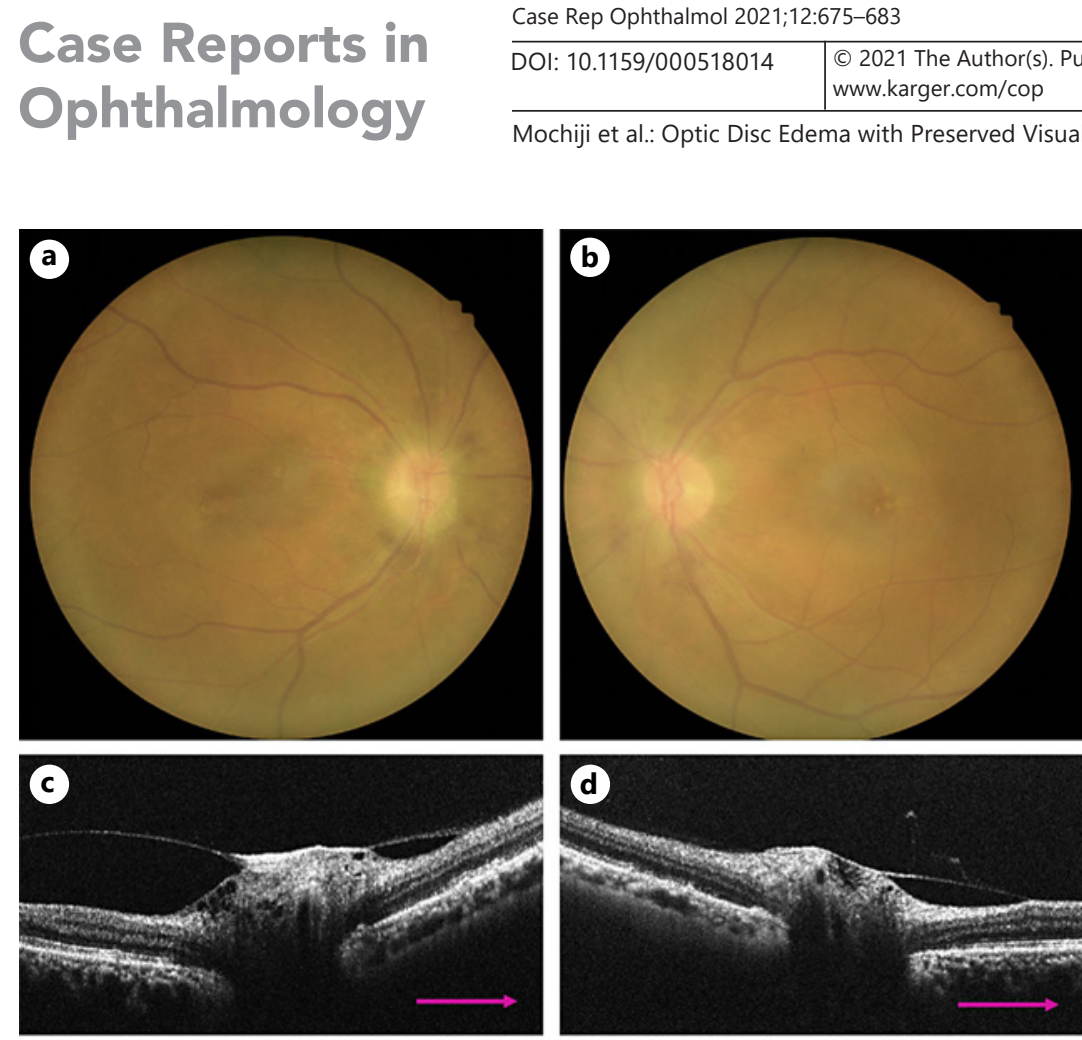

Right eye

Fig. 6. Fundus photographs (a, b) and OCT scans (c, d) at 4 months after the start of steroid treatment. Compared to the initial visit, optic disc swelling is improved, and retinal hemorrhage is reduced in his both eyes. Pink arrows indicate OCT scan directions. OCT, optical coherence tomography.

peared. Compared to the initial visit, ODS and retinal hemorrhage were improved OU (Fig. 6a-d). BCVA of 0.5 OD and $0.4 \mathrm{OS}$ and IOP of $12 \mathrm{~mm} \mathrm{Hg} 0 \mathrm{U}$ at the last visit were unchanged from those of his initial visit.

\section{Discussion/Conclusion}

In the current case, possible causes of papilledema including idiopathic intracranial hypertension, tumor, hemorrhage, venous sinus thrombosis, and granulomatous meningitis [3] were all denied by neuroimaging and lumbar puncture, and as a result, presence of GCA was diagnosed. In a retrospective review of consecutive 221 patients with bilateral ODS with preserved visual function, diagnosis other than papilledema was found only in $4(1.8 \%)$ patients [1]. Previously, bilateral ODS without visual disturbance was reported to be associated with nonarteritic AION, optic-perineuritis secondary to MPO-ANCA-positive vasculitis or neurosyphilis, intermediate uveitis, hypertensive emergency, and rapid improvement of glucose control in diabetes [1, 2, 4, 8]. Based on the European League Against Rheumatism recommendations for the use of imaging in large vessel vasculitis in clinical practice [9], since the findings of FDG-PET, enhanced CT, and enhanced MRI were highly suspicious of GCA, we have made the diagnosis of GCA without biopsy of the temporal artery. Accordingly, to the best of our knowledge, bilateral ODS with preserved visual function from GCA is unique in the literature.

GCA, although categorized as a large vessel vasculitis, is known to affect smaller-sized vessels and associates with a wide variety of ocular signs including arteritic AION, posterior ischemic optic neuropathy, central retinal artery occlusion, pupil involvement, ophthalmoplegia, scleritis, peripheral ulcerative keratitis, and neovascular glaucoma, although the arte- 
ritic AION is by far the most common ocular manifestation $[5,6]$. The fundus findings in our case including whitening/sheathing of arterioles in the peripapillary region (Fig. 4) seem similar with the fundus findings of a nonarteritic AION with preserved vision case in the previous report [1], and thus the ischemic mechanism might associate with the ODS in our case. The floaters reported by this case might be explained by the secondary phenomenon to the disturbed blood flow to the optic nerve/retina due to ODS. In the current case, none of the typical signs of AION such as pallid optic nerve swelling, filling defect of the optic nerve head during fluorescein angiography, visual field defect, or decreased visual acuity was observed. Although enhancement of the optic nerve sheath was not observed by the orbital MRI, inflammation can be another explanation of perineuritis-like findings [1] of our case.

Although rare, bilateral ODS with no visual disturbance can occur in patients without elevation of ICP. Reviewing of fundus photographs was the critical step to forward the differential diagnosis in this case; thus, we learnt from the case the importance of careful assessment of ocular findings to reach correct diagnosis of underlying systemic disease such as GCA.

\section{Statement of Ethics}

This study adhered to the tenets of the Declaration of Helsinki. The Institutional Review Board of Shimane University Hospital did not require an ethics committee review process to report this case. The patient provided written informed consent for publication of this case report and any accompanying pictures.

\section{Conflict of Interest Statement}

The authors have no conflicts of interest for this study.

\section{Funding Sources}

No financial support was provided.

\section{Author Contributions}

M.M., Y.Y., A.I., M.H., Y.M., and M.T. treated the subject and collected the clinical data. M.M. and M.T. wrote the manuscript, and Y.Y., A.I., M.H., and Y.M. revised the manuscript. All authors approved the final version of the manuscript. The authors agree to be responsible for all aspects of this work.

\section{Data Availability Statement}

All data generated or analyzed during this study are included in this article. Further enquiries can be directed to the corresponding author. 


\section{References}

1 Yu CW, Micieli JA. Bilateral optic disc edema with preserved visual function not related to papilledema. J Neurol Sci. 2020;418:117160.

2 Almog Y, Goldstein M. Visual outcome in eyes with asymptomatic optic disc edema. J Neuroophthalmol. 2003; 23(3):204-7.

3 Crum OM, Kilgore KP, Sharma R, Lee MS, Spiegel MR, McClelland CM, et al. Etiology of papilledema in patients in the eye clinic setting. JAMA Netw Open. 2020;3(6):e206625.

4 Parker SE, Pula JH. Neurosyphilis presenting as asymptomatic optic perineuritis. Case Rep Ophthalmol Med. 2012;2012:621872.

5 De Smit E, O’Sullivan E, Mackey DA, Hewitt AW. Giant cell arteritis: ophthalmic manifestations of a systemic disease. Graefes Arch Clin Exp Ophthalmol. 2016;254(12):2291-306.

6 Vodopivec I, Rizzo JF 3rd. Ophthalmic manifestations of giant cell arteritis. Rheumatology. 2018;57(Suppl 2): ii63-72.

7 Bloch DA, Michel BA, Hunder GG, McShane DJ, Arend WP, Calabrese LH, et al. The American College of Rheumatology 1990 criteria for the classification of vasculitis. Patients and methods. Arthritis Rheum. 1990;33(8): 1068-73.

8 Agardh CD, Cavallin-Sjöberg U, Agardh E. Optic disc swelling in an insulin-dependent diabetic. A result of drastic improvement of glucose control? Acta Ophthalmol. 1988;66(2):206-9.

9 Dejaco C, Ramiro S, Duftner C, Besson FL, Bley TA, Blockmans D, et al. EULAR recommendations for the use of imaging in large vessel vasculitis in clinical practice. Ann Rheum Dis. 2018;77(5):636-43. 